\title{
Three-Body Kick to a Bright Quasar Out of Its Galaxy during a Merger
}

\section{Citation}

Hoffman, Loren, and Abraham Loeb. 2006. “Three-Body Kick to a Bright Quasar Out of Its Galaxy during a Merger." The Astrophysical Journal 638 (2): L75-78. https://doi.org/10.1086/501230.

\section{Permanent link}

http://nrs.harvard.edu/urn-3:HUL.InstRepos:41393455

\section{Terms of Use}

This article was downloaded from Harvard University's DASH repository, and is made available under the terms and conditions applicable to Other Posted Material, as set forth at http:// nrs.harvard.edu/urn-3:HUL.InstRepos:dash.current.terms-of-use\#LAA

\section{Share Your Story}

The Harvard community has made this article openly available.

Please share how this access benefits you. Submit a story.

\section{Accessibility}




\title{
THREE-BODY KICK TO A BRIGHT QUASAR OUT OF ITS GALAXY DURING A MERGER
}

\author{
LOREN HoFFman ${ }^{1}$ AND ABRAham LoeB ${ }^{2}$ \\ Received 2005 December 12; accepted 2006 January 13; published 2006 February 6
}

\begin{abstract}
The quasar HE 0450-2958 was recently discovered to reside $\sim 7 \mathrm{kpc}$ away from a galaxy that was likely disturbed by a recent merger. The lack of a massive spheroid of stars around the quasar raised the unlikely suggestion that it may have formed in a dark galaxy. Here we explain this discovery as a natural consequence of a dynamical kick imparted to the quasar as it interacted with a binary black hole system during a galaxy merger event. The typical binary stalling radius provides a kick on the order of the escape velocity of the stellar spheroid, bringing the quasar out to around the observed radius before it reverses direction. This is consistent with the observed low relative velocity between the quasar and the merger-remnant galaxy. The gas carried with the black hole throughout the three-body interaction fuels the quasar for the duration of its journey, $\sim 2 \times 10^{7}$ years. Gravitational radiation recoil could not have produced the required kick.

Subject headings: black hole physics — galaxies: evolution — galaxies: interactions — galaxies: nuclei — galaxies: starburst — quasars: individual (HE 0450-2958)

Online material: color figures
\end{abstract}

\section{INTRODUCTION}

Observations indicate that every massive galaxy contains a supermassive black hole in its nucleus, which shines as a quasar during episodes of rapid mass accretion. The vigorous gas inflows needed to fuel the most luminous quasars are thought to be triggered by galaxy mergers, in which tidal interactions channel large amounts of gas into the central $\sim 100 \mathrm{pc}$ (Byrd et al. 1987; Hernquist 1989). The maximum luminosities of quasars are strongly correlated with the properties of their host galaxies (Shields et al. 2003; Floyd et al. 2004), in accordance with the tight correlations observed between black hole mass and stellar bulge mass (Magorrian et al. 1998; Marconi \& Hunt 2003; Peng et al. 2006) and velocity dispersion (Ferrarese \& Merritt 2000; Gebhardt et al. 2000; Tremaine et al. 2002) in quiescent galaxies.

Given these correlations, the recent discovery (Magain et al. $2005)$ of a bright $\left(M_{V}=-25.8\right)$ quasar at redshift $z=0.285$ apparently not surrounded by a massive host galaxy is surprising indeed. For emission at the Eddington luminosity, the inferred black hole mass is $\sim 4 \times 10^{8} M_{\odot}$. There is a neighboring, luminous starburst galaxy at a separation of $\sim 1$.'7, corresponding to $7.2 \mathrm{kpc}$ for a flat universe with a present-day Hubble parameter $H_{0}=71 \mathrm{~km} \mathrm{~s}^{-1} \mathrm{Mpc}^{-1}$ and a matter density parameter $\Omega_{m}=0.27$. This companion galaxy has a disturbed morphology and is heavily obscured by dust, typical of the ultraluminous infrared galaxies (ULIRGs) systematically associated with near-equal-mass mergers (Sanders et al. 1988). Its visual magnitude is $M_{V}=-23$. Close to the quasar lies a bright blob about $2 \mathrm{kpc}$ across, consisting of gas ionized by the quasar radiation. The spectrum of the blob shows no stellar component and no dust extinction; hence the host galaxy cannot be hidden by dust. The typical host for an $M_{V}=-25.8$ quasar is an elliptical galaxy with $M_{V} \approx-23.2$, about the magnitude of the neighboring ULIRG. However, after carefully deconvolving a new Hubble Space Telescope image of the system,

\footnotetext{
${ }^{1}$ Department of Physics, Harvard University, 17 Oxford Street, Cambridge, MA 02138; lhoffman@cfa.harvard.edu.

${ }^{2}$ Department of Astronomy, Harvard University, 60 Garden Street, Cambridge, MA 02138; aloeb@cfa.harvard.edu.
}

Magain et al. (2005) find no sign of any such galaxy surrounding this quasar. Fixing the half-light radius at $R_{e}=10 \mathrm{kpc}$ (a typical size for the host of an $M_{V} \sim-26$ quasar), they place a conservative upper limit of $M_{V}=-21.2,3.7 \sigma$ fainter than expected, on the magnitude of any host. Alternatively, adopting $M_{V}=-23$ for the host magnitude, they set an upper limit of $\sim 100 \mathrm{pc}$ on $R_{e}$, much too small for a normal galaxy of this magnitude.

We suggest that the quasar observed in HE 0450-2958 may be a black hole that was ejected from the nucleus of the companion galaxy during the same major merger that caused its disturbed appearance. The gas now fueling the quasar was dragged with it from the galactic center when it was kicked out. If one of the merging galaxies harbored a binary black hole, then when the black hole from the other galaxy entered the system, a complex three-body interaction would ensue, shortly ending with the ejection of the lightest black hole at a velocity on the order of the binary orbital velocity (Heggie 1975). This scenario implies that one of the merging galaxies was relatively gas-poor (perhaps a giant elliptical galaxy), to allow survival of the binary against gasdynamical friction for a Hubble time before the merger. On the other hand, at least one of the galaxies must have been gas-rich, in order to fuel the quasar. The two galaxies must have been close in mass, to explain the ULIRG appearance of the merger remnant and the high inferred black hole mass of the quasar, since it is the lightest body that gets ejected.

The Eddington accretion rate for a black hole of mass $M=$ $4 \times 10^{8} M_{\odot}$ is $\dot{M}_{\mathrm{Edd}}=\left(4 \pi G M m_{p}\right) /\left(\epsilon \sigma_{\mathrm{T}} c\right)=8.9 \epsilon_{-1}^{-1} M_{\odot} \mathrm{yr}^{-1}$, where $m_{p}$ is the proton mass, $\sigma_{\mathrm{T}}$ is the Thomson cross section, and $\epsilon=0.1 \epsilon_{-1}$ is the radiative efficiency. It takes $\sim 2 \times 10^{7} \mathrm{yr}$ for the black hole to reach a turnaround radius of $7.2 \mathrm{kpc}$, so in order to remain shining with $\epsilon_{-1} \sim 1$ at the observed distance, the black hole must drag at least $\sim 36 \%$ of its own mass with it in gas when it is ejected. The distances of closest approach in our three-body interactions are often larger than the radius containing this mass in an $\alpha$-disk (Shapiro \& Teukolsky 1983), and so the inner accretion disk can follow the black hole throughout the interaction and get ejected with it. 


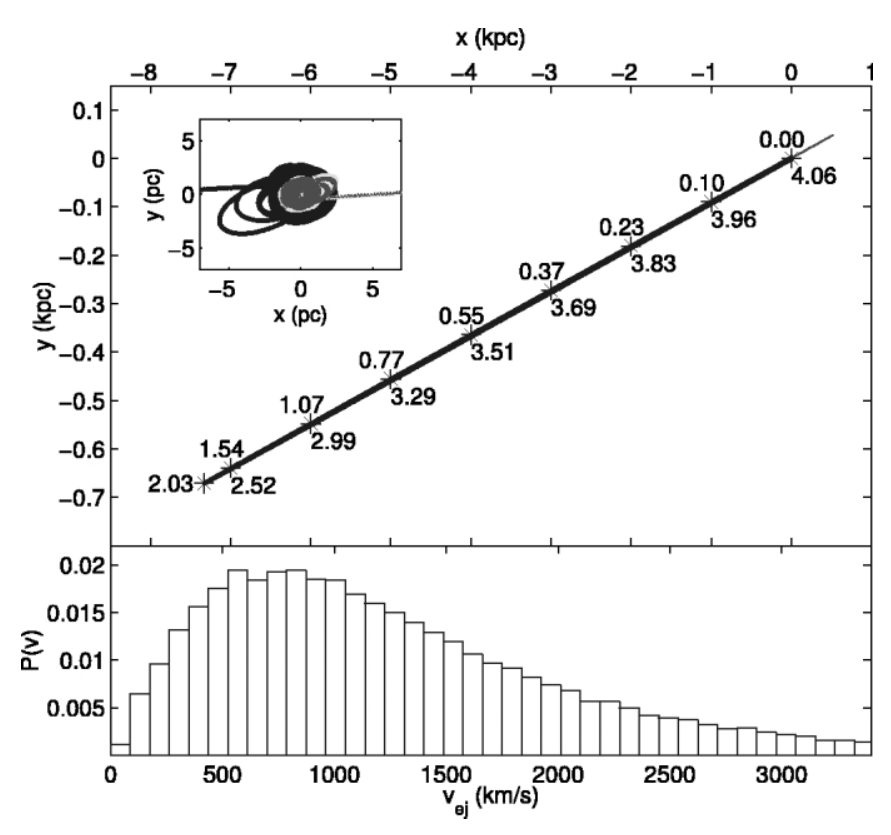

FIG. 1.-Top: Black hole trajectories following a three-body ejection. The thick line shows the path of the intruder, and the thin lines that of the binary members; each is plotted only until its first return to the galactic center. Time labels in units of $10^{7} \mathrm{yr}$ are marked off at various points along the trajectory, going out and returning. In the inset we zoom in on the innermost $\pm 7 \mathrm{pc}$. Here the trajectories are plotted only up to the point of ejection (with return trajectories left out). One can clearly see the binary and intruder flying off in opposite directions at the end of the interaction. Bottom: The distribution of ejection velocities over all $\sim 10^{5}$ runs. [See the electronic edition of the Journal for a color version of this figure.]

\section{BLACK HOLE BINARIES AND EJECTIONS}

When two galaxies of comparable mass merge, their black holes sink to the center by dynamical friction and form a gravitationally bound binary. Once the binary separation reaches the "hardening radius," $r_{\text {hard }} \approx G\left[\min \left(M_{1}, M_{2}\right)\right] / 4 \sigma^{2} \sim 1-5 \mathrm{pc}$ for massive galaxies (where $\sigma$ is the one-dimensional stellar velocity dispersion and $M_{1}$ and $M_{2}$ are the black hole masses), its further reduction is dominated by close encounters with stars that pass within the binary orbit. These stars undergo strong three-body interactions with the binary and escape its vicinity with speeds comparable to the binary orbital speed. Only stars on nearly radial orbits can contribute to the hardening of the binary in this stage. In the low-density nuclei of massive elliptical galaxies, the total mass in stars on these so-called losscone orbits is small compared with the mass of the binary. Since the two-body relaxation time is long compared with the Hubble time, stars cannot diffuse back into the loss cone as fast as they are kicked out. If all stars on loss cone orbits run out, the decay of the binary ceases. Since the binary ejects of order its own mass in stars per $e$-folding in radius, one naively would expect binaries in the cores of massive elliptical galaxies to stall at separations on the order of $1 \mathrm{pc}$. If the separation reaches $\sim 0.01-0.1 \mathrm{pc}$, then the binary will quickly coalesce through the emission of gravitational radiation. The question of whether and how it crosses the gap from $\sim 1 \mathrm{pc}$ to this smaller scale is known as the "final parsec problem" (Merritt \& Milosavljević 2005).

Black hole binaries may coalesce in some cases but not others. For example, the mass of any close binary companion to the nuclear black hole of the Milky Way is limited to $M_{2}$ $\lesssim 10^{4} M_{\odot}$ by the Keplerian orbits of stars near the Galactic center (Merritt \& Milosavljević 2005). The so-called X-shaped radio galaxies have been explained as jets bent by sudden changes in the spin of a black hole upon coalescence with a binary companion (Merritt \& Milosavljević 2005). Gas drag may lead to coalescence in gas-rich mergers (Merritt \& Milosavljević 2005); however, the gas-poor cores of massive elliptical galaxies could allow binaries to stall. There are some tentative observational hints that subparsec-scale binaries exist, including periodic variations and double-peaked broad-line profiles in active galaxies, and central minima in the surface brightness profiles of some early-type galaxies, which may be associated with the clearing of the loss cone around stalled binaries (Komossa 2003).

When an unequal-mass black hole binary coalesces by gravitational radiation, the waves carry off a net linear momentum and the merged black hole recoils in the opposite direction (Favata et al. 2004). However, $300 \mathrm{~km} \mathrm{~s}^{-1}$ is a reasonable upper limit to the velocity achievable by this mechanism (Merritt et al. 2004; Blanchet et al. 2005). A mass ejected radially at $300 \mathrm{~km} \mathrm{~s}^{-1}$ from the center of the average stellar spheroid hosting a $4 \times 10^{8} M_{\odot}$ black hole $\left(M_{\mathrm{vir}}=1.7 \times 10^{11} M_{\odot}, R_{e}=\right.$ $3.6 \mathrm{kpc}$ ) would only reach a distance of $\sim 150 \mathrm{pc}$ before turning around, far smaller than the separation of the quasar in $\mathrm{HE}$ 0450-2958 from its companion galaxy.

If a binary stalls and its host galaxy merges with another galaxy, a third black hole may spiral in and undergo strong three-body interactions with the pair. The merger event will drive mass through the binary at a significant rate, partially refilling the loss cone, but the final stages of the merger transpire so rapidly that the intruder is likely to arrive in the vicinity of the binary before the binary has a chance to coalesce (Milosavljević \& Merritt 2001). The three-body interaction will end in the hardening of the binary by an amount comparable to its initial binding energy, and the ejection of the lightest body at a speed comparable to the binary's orbital speed (Heggie 1975). The binary's orbital speed at the stalling radius is a few times $\sigma$, that is, on the order of the escape velocity $v_{\text {esc }}$ from the stellar bulge [e.g., for a Hernquist profile (Hernquist 1990) $v_{\text {esc }}=$ $2 \sqrt{2} v_{\text {circ }}(r=a)$ with $\left.\sigma \sim v_{\text {circ }}\right]$. Thus, qualitatively we expect three-body kicks to yield ejection velocities on the order of the bulge escape velocity, resulting in many near-ejections. This is consistent with the large distance and low velocity of the HE 0450-2958 quasar relative to the ULIRG. Figure 1 shows an example of such a near-escape trajectory.

\section{NUMERICAL CALCULATIONS}

To make our dynamical arguments more quantitative, we ran a series of 83,250 three-body scattering experiments using S. Aarseth's K-S-regularized (Kustaanheimo \& Stiefel 1965) Chain code (Aarseth 2003) to compute the black hole trajectories. In each case the intruder's mass was set to $M_{3}=4 \times$ $10^{8} M_{\odot}$ and the masses of the initial binary members were chosen uniformly in their logarithm between 1.0 and 3.5 times the mass of the intruder, a generic range for major mergers. From here on we will refer to the initial black hole binary as the inner binary and the "binary" formed by the intruder and the inner binary's center of mass as the outer binary. The semimajor axis $a_{\text {bin }}$ of the inner binary was always initialized to $r_{\text {hard }} / 4$, and that of the outer binary was set using the stability criterion of Mardling \& Aarseth $(1999),\left(R_{p}^{\text {out }} / a_{\text {in }}\right) \approx 2.8[(1+$ $\left.\left.q_{\text {out }}\right)\left(1+e_{\text {out }}\right) /\left(1-e_{\text {out }}\right)^{1 / 2}\right]^{2 / 5}$, which reliably estimates the most distant intruder orbit for which strong three-body interactions can begin. Here $R_{p}^{\text {out }}$ is the pericenter distance of the outer binary, $a_{\text {in }}$ is the semimajor axis of the inner binary, $q_{\text {out }}=$ 
$M_{3} /\left(M_{1}+M_{2}\right)$ is the mass ratio of the outer binary, and $e_{\text {out }}$ is the outer binary's eccentricity. The initial eccentricity of the inner and outer binaries was chosen uniformly between 0.0 and 0.2 and 0.3 , respectively, where this range of low eccentricities naturally results from dynamical friction. Finally, the three Euler angles of the intruder's orbital plane were chosen randomly relative to the reference plane of the binary orbit, as was the phase of the initial periastron of the binary. Both orbits were always started at pericenter; since many orbital periods elapse before unstable interactions begin, the relative phase is effectively randomized in any case.

The Chain code allows the user to add conservative or velocity-dependent external perturbations, and so we added a dynamical friction force given by Chandrasekhar's formula (Chandrasekhar 1943) to the motion of each body. The density and Coulomb logarithm $\ln \Lambda$ were chosen to give a dynamical friction timescale $\sim 10^{6} \mathrm{yr}$. Each run was terminated when (1) any one of the bodies was expelled to a distance of $1 \mathrm{kpc}$ from the center of mass; (2) any two bodies came within $30 r_{\mathrm{S}}$ (where $r_{\mathrm{S}}=2 G M / c^{2}$ is the Schwarzschild radius) of each other or the gravitational radiation timescale dropped below $10^{5} \mathrm{yr}$ (roughly the dynamical time) for any pair; or (3) the total time elapsed reached $6 \times 10^{6} \mathrm{yr}$. We selected out just those runs where the intruder never came within 5000 Schwarzschild radii $(\sim 0.2 \mathrm{pc})$ of either binary member, reflecting the scale below which an $\alpha$-disk (Shapiro \& Teukolsky 1983) with sufficient mass to fuel the quasar might be disrupted. This is also about the size inferred for the broad-line region of the HE 0450-2958 quasar (Greene \& Ho 2005; Merritt et al. 2005). Of the runs not terminated by criterion 2 or 3 , the closest approach of the intruder was greater than $5 \times 10^{3} r_{\mathrm{S}}$ in $36 \%$ and greater than $10^{4} r_{\mathrm{s}}$ in $22 \%$ of the runs. To each successful run we then assigned a galaxy based on the empirical black hole/bulge correlations. Each galaxy was modeled as a Hernquist profile (Hernquist 1990) with a bulge virial mass (Marconi \& Hunt 2003) $M_{\mathrm{vir}} \approx 3 R_{e} \sigma^{2} / G$ chosen according to a lognormal distribution with a mean given by the relation $M_{\mathrm{vir}} \approx(1.71 \times$ $\left.10^{11} M_{\odot}\right)\left[M /\left(4 \times 10^{8} M_{\odot}\right)\right]^{1.04}$ and standard deviation of $0.25 \mathrm{dex}$ (Marconi \& Hunt 2003). The velocity dispersion was calculated from the $M-\sigma$ relation (Tremaine et al. 2002), $\sigma \approx$ $\left(262 \mathrm{~km} \mathrm{~s}^{-1}\right)\left[M /\left(4 \times 10^{8} M_{\odot}\right)\right]^{0.249}$, and the bulge scale radius set by $R_{e}=G M_{\mathrm{vir}} / 3 \sigma^{2}$. We then added to the stellar bulge a dark matter halo with a mass ratio that is an order of magnitude larger than the cosmic matter-to-baryon ratio (corresponding to a star formation efficiency of $10 \%$ ) and a concentration parameter of $9.0\left(M_{\text {halo }} / 2.10 \times 10^{13} M_{\odot}\right)^{-0.13}$ with a scatter of 0.18 dex (Bullock et al. 2001).

The black hole was ejected radially from the galactic center with the final velocity from the three-body run and its trajectory integrated for $6 \times 10^{7} \mathrm{yr}$. We imposed dynamical friction with $\ln \Lambda=3$, which had almost no effect on the trajectories in high-velocity ejections but efficiently damped out those with low velocities. Finally, we chose a random line of sight and projected all distances and velocities accordingly. We added up the total time spent in each logarithmic distance and velocity bin to assess the relative probability of seeing the quasar at different projected separations and line-of-sight velocities. Figure 2 shows the resulting probability distributions. The observed parameters of the quasar under consideration, $D \sim$ $7.2 \mathrm{kpc}$ and $v \lesssim 200 \mathrm{~km} \mathrm{~s}^{-1}$, appear to be fairly typical in the statistics of our runs. Note that the results scale simply with the black hole mass in our model. If the inner binary always starts at a fixed fraction of the hardening radius, then $M_{\mathrm{bh}} \propto$ $\sigma^{4}$ gives $a_{\mathrm{bin}} \propto M_{\mathrm{bh}} / \sigma^{2} \propto M_{\mathrm{bh}}^{1 / 2}$. We then have $v_{\mathrm{ej}} \propto\left(M_{\mathrm{bh}} / a_{\mathrm{bin}}\right)^{1 / 2}$

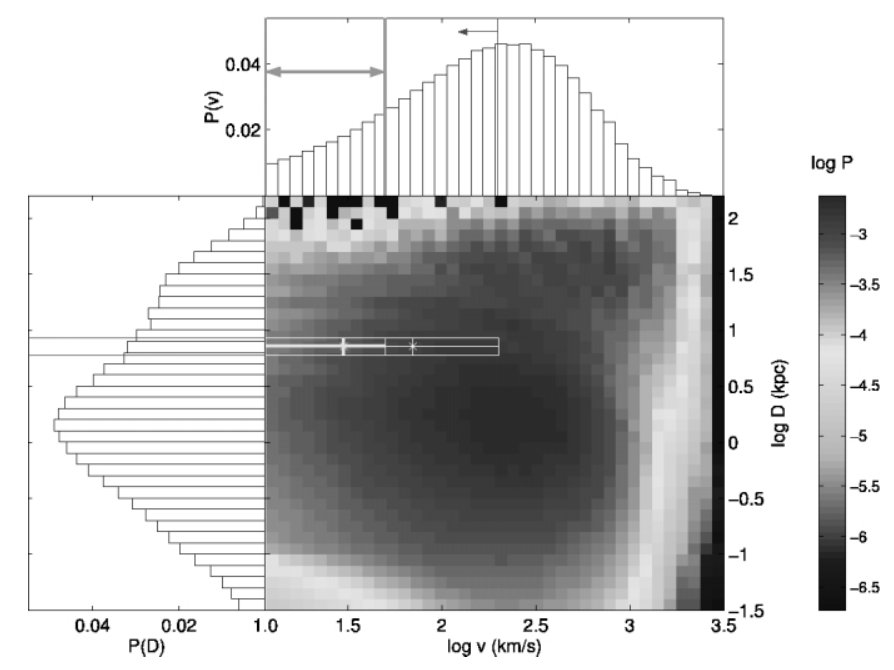

FIG. 2.-Probability $P$ of observing the black hole at various projected separations $D$ and line-of-sight velocities $v$. The gray scale is logarithmic in $P(D, v)$, where $P(D, v)$ is the probability density for obtaining the values $(D, v)$. The probability incorporates the length of time spent by the ejected black hole at these values, averaged over $\sim 10^{4}$ successful runs (which were neither terminated before the ejection nor eliminated by our minimum-distance criterion). The white star shows the estimated coordinates of the HE 04502958 quasar based on the radial velocity range reported by Magain et al., with errors bracketed by the white lines. The thicker lines indicate the magnitude of the quasar's redshift relative to the galaxy $\left(30 \pm 20 \mathrm{~km} \mathrm{~s}^{-1}\right)$ measured by Merritt et al. (2005). The histograms show the corresponding projections of $P$ onto the $D$ - and $v$-axes. [See the electronic edition of the Journal for a color version of this figure.]

$\propto M_{\mathrm{bh}}^{1 / 4} \propto \sigma$. The self-similarity of the Hernquist model implies that the turnaround distance $r_{\max }$ in units of the galactic scale radius is fixed by $v_{\text {ej }}$ in units of $\sigma$. Since $v_{\text {ej }} / \sigma$ is constant, we get $r_{\max } \propto R_{e} \propto M_{\mathrm{bh}}^{1 / 2}$. Hence neglecting minor deviations from self-similarity due to dynamical friction, we expect the velocities in our plots to scale roughly as $M_{\mathrm{bh}}^{1 / 4}$ and distances as $M_{\mathrm{bh}}^{1 / 2}$.

\section{DISCUSSION AND CONCLUSIONS}

We have proposed that the HE 0450-2958 quasar, around which no galactic host has so far been detected, was ejected from the nearby galaxy in a three-body interaction following a merger of a massive gas-rich galaxy with a giant elliptical galaxy. This scenario explains (1) the fueling of the bright quasar displaced by $\sim 7 \mathrm{kpc}$ from the galactic center, (2) the survival of the binary in the gas-poor galaxy before the merger, and (3) the disturbed appearance of the companion galaxy. One may also ask how the presence of the "blob" and narrow line emission in the quasar spectrum, both indicative of ionized gas in the quasar's vicinity far outside the black hole radius of influence, fit into the ejection picture. Merritt et al. (2005) infer a size of roughly $1.5 \mathrm{kpc}$ for the narrow-line region (NLR). Since the gas at this distance cannot possibly be bound to the black hole, they argue that the very small observed redshifts between quasar, galaxy, and narrow emission line gas rule out the ejection scenario. However, since the ejected quasar would spend most of its time near turnaround, we find a quite small velocity difference between the quasar and ULIRG to be consistent with the high-velocity ejection model. For instance, the black hole modeled in Figure 1, with $v_{\text {ej }} \sim 1200 \mathrm{~km} \mathrm{~s}^{-1}$, spends $17 \%$ of its time with $v<100 \mathrm{~km} \mathrm{~s}^{-1}$. After projecting onto the line of sight and averaging over many runs as in Figure 2, one 
can see that very small velocities are still prevalent in our statistics at distances around $7 \mathrm{kpc}$.

In galaxy merger simulations including feedback from star formation and active galactic nuclei (Springel et al. 2005), large amounts of gas are blown from the galactic center out to distances of $\sim 5-10 \mathrm{kpc}$ during the coalescence of the two nuclei. The disturbed system also shines as a ULIRG during most of this phase. Once the feedback shuts off, the gas that does not escape settles back into the inner galaxy on the dynamical timescale of $\sim 10^{8}$ yr. The mass in narrow-line-emitting gas inferred from absolute line fluxes in typical NLR is around $10^{5}-10^{6} M_{\odot}$ (Peterson 1997). Ten million years after the core merger, more than this mass in gas below $10^{5} \mathrm{~K}$ would likely be available within the few-kiloparsec sphere surrounding a black hole ejected to a distance of $\sim 7 \mathrm{kpc}$. It is plausible that clouds formed from this cool gas could produce the observed narrow line emission in the quasar spectrum while the black hole (and gas) are near turnaround. The observed blob would be a portion of the gas photoionized by anisotropic radiation from the quasar. It is not feasible for Bondi accretion from this external gas to fuel the quasar, as the densities required are unrealistically high. Rather, the quasar must be fueled by the remnant inner accretion disk dragged with it from the galactic center, which can remain bound to it through the ejection as demonstrated above.

Our proposed scenario is viable as long as the merger process does not induce coalescence of the binary through dynamical friction on a rearranged distribution of stars and gas, before the three black holes interact. For typical parameters, we find that the feeding of the loss cone of stars by tidal interactions during the merger is not sufficient to cause coalescence of the binary before the third black hole sinks in (Roos 1981). Coalescence through friction on gas would be possible by momentum conservation only if a large quantity of gas, comparable to or larger than the binary mass, were channeled through the interior of the binary before the three black holes interact. However, since the gas initially resides within the quasar's original host galaxy, it is more likely to fuel the quasar before reaching the nucleus of the dry elliptical galaxy that hosts the binary. Feedback from the quasar could then easily supply sufficient energy to prevent the gas that gets tidally stripped from the outer envelope of its host galaxy (Wyithe \& Loeb 2003) from settling into the few-parsec-scale region surrounding the binary before the three black holes interact.

Both our calculations and the identification of HE 04502958 as a quasar without a typical host (Magain et al. 2005) are based on the correlations between black hole masses and their host bulges established for isolated, quiescent galaxies. The extension of these correlations to active and merging systems is somewhat less certain. Several authors (Greene \& Ho 2006; Onken et al. 2004; Wandel 2002) have studied the $M_{\mathrm{bh}}-\sigma$ and $M_{\mathrm{bh}}-M_{\text {bulge }}$ relations in active galaxies and reported statistically significant deviations from the slope and zero point measured for inactive systems, as well as slightly larger scatter. However, the reported deviations are not typically greater than a factor of 2-3 and hence qualitatively support our application of the correlations to an interacting system.

Since the mass of the HE 0450-2958 black hole and corresponding expected host properties are uncertain, one cannot prematurely conclude that it is truly a "naked" quasar. Further observations, particularly in bands admitting a higher ratio of galaxy to quasar luminosity, are needed to place tighter constraints on the properties of any host galaxy. This Letter demonstrates the plausibility of a three-body ejection producing a naked quasar near a recently merged galaxy. If such a system is unambiguously observed, this would provide a key link in our understanding of galaxy formation and the hierarchical buildup of supermassive black holes.

We are grateful to Sverre Aarseth for use of his Chain code and for enlightening discussions about black hole binaries. We also thank Thomas J. Cox, Volker Springel, and Lars Hernquist for providing simulation data and for useful discussions about the coevolution of black holes and galaxies. This work was supported in part by NASA grants NAG 5-13292 and NNG05GH54G, and NSF grant AST 02-04514.

\section{REFERENCES}

Aarseth, S. 2003, Gravitational N-Body Simulations: Tools and Algorithms (Cambridge: Cambridge Univ. Press)

Blanchet, L., Qusailah, M. S. S., \& Will, C. M. 2005, ApJ, 635, 508

Bullock, J. S., Kolatt, T. S., Sigad, Y., Somerville, R. S., Kravtsov, A. V., Klypin, A. A., Primack, J. R., \& Dekel, A. 2001, MNRAS, 321, 559

Byrd, G. G., Sundelius, B., \& Valtonen, M. 1987, A\&A, 171, 16

Chandrasekhar, S. 1943, ApJ, 97, 255

Favata, M., Hughes, S. A., \& Holz, D. E. 2004, ApJ, 607, L5

Ferrarese, L., \& Merritt, D. 2000, ApJ, 539, L9

Floyd, D. J. E., Kukula, M. J., Dunlop, J. S., McLure, R. J., Miller, L., Percival, W. J., Baum, S. A., \& O’Dea, C. P. 2004, MNRAS, 355, 196 Gebhardt, K., et al. 2000, ApJ, 539, L13 (erratum 555, L75 [2001])

Greene, J. E., \& Ho, L. C. 2005, ApJ, 630, 122 2006, ApJL, in press (astro-ph/0512461)

Heggie, D. C. 1975 , MNRAS, 173, 729

Hernquist, L. 1989, Nature, 340, 687 . 1990, ApJ, 356, 359

Komossa, S. 2003, in AIP Conf. Proc. 686, The Astrophysics of Gravitational Wave Sources, ed. J. M. Centrella (Melville, NY: AIP), 161

Kustaanheimo, P., \& Stiefel, E. L. 1965, J. Reine Angew. Math., 218, 204

Magain, P., Letawe, G., Courbin, F., Jablonka, P., Jahnke, K., Meylan, G., \& Wisotzki, L. 2005, Nature, 437, 381

Magorrian, J., et al. 1998, AJ, 115, 2285

Marconi, A., \& Hunt, L. K. 2003, ApJ, 589, L21
Mardling, R., \& Aarseth, S. 1999, in The Dynamics of Small Bodies in the Solar System, ed. B. A. Steves \& A. E. Roy (NASO ASI Ser. C, 522) (Boston: Kluwer), 385

Merritt, D., Storchi Bergmann, T., Robinson, A., Batcheldor, D., Axon, D., \& Cid Fernandes, R. 2005, MNRAS, submitted (astro-ph/0511315)

Merritt, D., \& Milosavljević, M. 2005, Living Rev. Relativ., 8, 8

Merritt, D., Milosavljević, M., Favata, M., Hughs, S. A., \& Holz, D. E. 2004, ApJ, 607, L9

Milosavljević, M., \& Merritt, D. 2001, ApJ, 563, 34

Onken, C. A., Ferrarese, L., Merritt, D., Peterson, B. M., Pogge, R. W., Vestergaard, M., \& Wandel, A. 2004, ApJ, 615, 645

Peng, C. Y., Impey, C. D., Ho, L. C., Barton, E. J., \& Rix, H.-W. 2006, ApJ, in press (astro-ph/0509155)

Peterson, B. 1997, An Introduction to Active Galactic Nuclei (Cambridge: Cambridge Univ. Press)

Roos, N. 1981, A\&A, 104, 218

Sanders, D. B., Soifer, B. T., Elias, J. H., Madore, B. F., Matthews, K., Neugebauer, G., \& Scoville, N. Z. 1988, ApJ, 325, 74

Shapiro, S. L., \& Teukolsky, S. A. 1983, Black Holes, White Dwarfs, and Neutron Stars (New York: Wiley)

Shields, G. A., Gebhardt, K., Salviander, S., Wills, B. J., Xie, B., Brotherton, M. S., Yuan, J., \& Dietrich, M. 2003, ApJ, 583, 124

Springel, V., Di Matteo, T., \& Hernquist, L. 2005, MNRAS, 361, 776

Tremaine, S., et al. 2002, ApJ, 574, 740

Wandel, A. 2002, ApJ, 565, 762

Wyithe, J. S. B., \& Loeb, A. 2003, ApJ, 595, 614 